\title{
SYNTHESIS OF ETHYLENE WITH ACREMONIUM SP. 502 PHYTOPATHOGENIC FUNGI
}

\author{
G. V. Tsehmister
}

Representatives of Cucurbitaceae family are among the most common types of vegetables grown in Ukraine. The problem of their destruction with fungi in Ukraine has not been investigated enough. Besides, pathogenic fungi lead to considerable losses in agriculture. Representatives of Acremonium Link genus, mostly lead saprotrophic way of nutrition, but in certain circumstances they can change specialization and cause diseases of plants of Cucurbitaceae family, manifesting themselves as facultative parasites. Fungi of Acremonium genus were isolated from diseased plants of melons in Spain, Italy and the US (California and Texas) [13-18; 20]. In 2011, S.P. Nadkernychnyi separated strain of Acremonium sp. 502 from cucumber plants were grown under conditions of closed ground and had disease symptoms. We have confirmed its pathogenicity on cucumber plants of Koroliok variety. It was revealed that it is localized in the root system, root neck and hypocotyl and the disease starts to manifest itself in the phase of true leaves. [12]

Phytopathogenic microorganisms produce a number of secondary metabolites that negatively affect the growth and development of plants. We know that the ability to synthesize phytohormones often play an important role in the pathogenesis and correlates with virulence and specialization of pathogen. In case of obligate parasitism the use of host plant regulatory systems by pathogen is an important combination factor. Phytohormones were detected in spores, culture liquid and mycelium of many pathogenic fungi. It is believed that the intensive production of hormones is associated with the implementation of phytopathogenic features such as tissue necrosis and their hydrolysis into simple compounds [2], it is also known that hypersynthesis of phytohormones unbalances hormonal system of plants and is the cause of many diseases [7]. The determination of fungi ability to produce phytohormonal substances enables to determine the mechanism of their influence on plant organism. One of the components of fungi pathogenesis is ethylene production [4].
Ethylene is a natural plant growth regulator, one of the main phytohormones. In the plant it controls a wide range of physiological processes, ripening of fruit and aging of tissues, seed germination, growth of cells by stretching, as well as it participates in plants response to various stressors. Typical processes that are activated by ethylene are accelerating leaves aging [9], stunting of stem growth in length, its thickening and horizontal growth [1]. At the same time ethylene can reduce polar auxin transportation, inhibit cell division, accelerate fruit falling, cause flowers aging, stun roots growth [19]. It is known that fungi of Fusarium, Penicillium, Verticillium, Mucor, Saccharomyces genera are able to synthesize ethylene [9].

In view of the above, the objective of our study was to investigate the ability of Acremonium sp. 502 to produce ethylene as one of the mechanisms of influence on plants.

Materials and methods. Pathogenic strain of fungi of Acremonium genus, was studied, which was isolated from diseased cucumber plants that were grown in the conditions of closed ground. Strain virulence was preapproved on cucumbers of Koroliok variety. The culture of fungus was kept on slant wort-agar (4 Balling degrees). The description of morphological and cultural characteristics was presented previously by us [10].

To study the synthesis of ethylene Acremonium sp. 502 was superficially cultured in glass vials on Chapek synthetic medium at the temperature of $26-28^{\circ} \mathrm{C} .10 \mathrm{ml}$ of medium were poured on into $30 \mathrm{ml}$ vials. Acremonium sp. 502 seeding material was obtained by flushing conidia and fungus hyphae pieces from slant wort-agar. Sowing was carried with spore suspension $\left(\mathrm{T}=1 \times 10^{6} \mathrm{CFU}\right)$ in the amount of $5 \%$ from the volume of culture medium. Measurement of ethylene was performed every 7 days over 7 weeks. At this vials were sealed for a day. The composition of air in the gas phase was analyzed on gas chromatograph Chrom-4 [4]. The composition of air in vials without fungus inoculums served as a control. Fungus mycelium 
was separated through filter paper, washed several times with distilled water and dried to constant weight at $105^{\circ} \mathrm{C}$. The calculation of ethylene in the sample was done according to the proposed method [6].

As the criterion for assessing of observed changes probability standard deviation was calculated [5].

Thus, we found that pathogenic to cucumber plants Acremonium sp. 502 fungus is able to produce ethylene. The highest level of biosynthesis was recorded after 5 weeks of cultivation, coinciding with the terms of the highest level of cellulases synthesis that are involved in the penetration of the fungus into the plant. We can therefore assume that ethylene synthesis plays a certain role in the mechanism of pathogenesis of cucumber plants by influencing the activity of cellulases and accelerating leaves aging. 